\title{
Ansiedade e autoestima associadas ao baixo desempenho escolar em estudantes com dislexia de desenvolvimento: uma revisão integrativa
}

Anxiety and self-esteem associated with poor school performance in students with developmental dyslexia: an integrative review

Ansiedad y autoestima asociadas con un bajo rendimiento escolar en estudiantes con dislexia del desarrollo: una revisión integradora

Mara Dantas Pereira

Mestranda na Universidade Federal de Sergipe, São Cristóvão, SE, Brasil

E-mail: maradantaspereira@gmail.com ORCID: https://orcid.org/0000-0002-5943-540X

Joilson Pereira da Silva

Professor doutor da Universidade Federal de Sergipe, São Cristóvão, SE, Brasil

E-mail: joilsonp@hotmail.com ORCID: https://orcid.org/0000-0001-9149-3020

Recebido em 29 de agosto de 2020

Aprovado em 16 de julho de 2021

Publicado em 31 de agosto de 2021

\section{RESUMO}

A dislexia de desenvolvimento é uma disfunção neurobiológica ocasionada por fatores de ordem cognitiva, hereditária e ambiental. Tal disfunção provocaria uma dificuldade específica, significativa e persistente, em distintos graus, na aprendizagem da decodificação, comprometendo os processos de compreensão leitora e escrita. Podendo, ainda, ocasionar o risco de desenvolvimento da ansiedade e da baixa autoestima, estando significativamente correlacionadas ao baixo desempenho escolar em estudantes com dislexia. A esse tema, objetivou-se investigar sobre ansiedade e autoestima associadas ao baixo desempenho escolar em estudantes com dislexia. Trata-se de uma revisão integrativa da literatura, realizada a partir da busca por publicações científicas indexadas nas bases de dados MEDLINE/PubMed, Europe PMC e ERIC. Os seguintes descritores foram utilizados: ansiedade, autoestima, dislexia de desenvolvimento, dificuldade de desenvolvimento de leitura, educação, estudantes e transtorno da leitura da dislexia do desenvolvimento. Ao final das buscas, foram selecionados 11 estudos para compor o presente artigo, nos idiomas inglês e espanhol, publicados entre os anos de 2009 a 2020. Mediante aos achados, dos estudos analisados foi possível perceber que os estudantes com dislexia apresentaram um perfil emocional-comportamental, caracterizado por menor autoestima e maior ansiedade em relação aos fatores emocionais, além de maiores problemas comportamentais que os estudantes sem dislexia. Conclui-se que é preciso um melhor gerenciamento das emoções e um fortalecimento da autoestima para que haja uma minimização do baixo desempenho escolar em estudantes com dislexia. Para tanto, apoiase na fundamental importância da ampliação de ações entre a Psicologia e a Educação, 
http://dx.doi.org/10.5902/1984686X54521

elaborando intervenções precoces relacionadas às dificuldades de leitura ao ajustamento psicossocial.

Palavras-chave: Ansiedade; baixo desempenho escolar; dislexia de desenvolvimento.

\section{ABSTRACT}

Developmental dyslexia is a neurobiological disorder caused by cognitive, hereditary and environmental factors. Such dysfunction would cause a specific, significant and persistent difficulty, in different degrees, in the learning of decoding, compromising the processes of reading and writing comprehension. It can also cause the risk of developing anxiety and low self-esteem, being significantly correlated with low school performance in students with dyslexia. This theme aimed to investigate anxiety and self-esteem associated with poor school performance in students with dyslexia. This is an integrative literature review, based on the search for scientific publications indexed in the MEDLINE/PubMed, Europe PMC and ERIC databases. The following descriptors were used: anxiety, self-esteem, developmental dyslexia, difficulty in reading development, education, students and developmental dyslexia reading disorder. At the end of the searches, 11 studies were selected to compose the present article, in English and Spanish, published between the years 2009 to 2020. Based on the findings, from the studies analyzed, it was possible to notice that students with dyslexia had an emotional-behavioral profile, characterized by lower self-esteem and greater anxiety in relation to emotional factors, in addition to greater behavioral problems than students without dyslexia. It is concluded that a better management of emotions and a strengthening of self-esteem is necessary in order to minimize the low academic performance of students with dyslexia. To this end, it is based on the fundamental importance of expanding actions between Psychology and Education, developing early interventions related to reading difficulties and psychosocial adjustment.

Keywords: Anxiety; low school performance; developmental dyslexia.

\section{RESUMEN}

La dislexia del desarrollo es un trastorno neurobiológico causado por factores cognitivos, hereditarios y ambientales. Tal disfunción provocaría una dificultad específica, significativa y persistente, en diferentes grados, en el aprendizaje de la decodificación, comprometiendo los procesos de comprensión lectora y escrita. También puede provocar el riesgo de desarrollar ansiedad y baja autoestima, estando significativamente correlacionado con un bajo rendimiento escolar en estudiantes con dislexia. Este tema tuvo como objetivo investigar la ansiedad y la autoestima asociadas con el bajo rendimiento escolar en estudiantes con dislexia. Se trata de una revisión integradora de la literatura, basada en la búsqueda de publicaciones científicas indexadas en las bases de datos MEDLINE/PubMed, Europe PMC y ERIC. Se utilizaron los siguientes descriptores: ansiedad, autoestima, dislexia del desarrollo, dificultad en el desarrollo de la lectura, educación, estudiantes y trastorno de la lectura con dislexia del desarrollo. Al finalizar las búsquedas, se seleccionaron 11 estudios para componer el presente artículo, en los idiomas inglés y español, publicados entre los años 2009 a 2020. Con base en los hallazgos, de los estudios analizados, se pudo notar que los estudiantes con dislexia tenían un perfil emocional- 
conductual, caracterizado por menor autoestima y mayor ansiedad en relación a factores emocionales, además de mayores problemas de conducta que los estudiantes sin dislexia. Se concluye que es necesario un mejor manejo de las emociones y un fortalecimiento de la autoestima para minimizar el bajo rendimiento académico de los estudiantes con dislexia. Para ello, se sustenta en la importancia fundamental de ampliar las acciones entre Psicología y Educación, desarrollando intervenciones tempranas relacionadas con las dificultades lectoras para la adaptación psicosocial.

Palabras clave: Ansiedad; bajo rendimiento escolar; dislexia del desarrollo.

\section{Introdução}

A dislexia de desenvolvimento (DD) teve seu reconhecimento pela Federação Mundial de Neurologia (FMN), em 1968, sendo definida como uma perturbação que se apresenta por intermédio de dificuldades na aprendizagem da leitura (SOUZA; SILVA; COUTINHO, 2019). E, de acordo com a Associação Brasileira de Dislexia (ABD), é um distúrbio de maior incidência nas salas de aula, que atinge entre 5\% e 17\% da população mundial (BRASIL, 2008).

Em 2002, a Associação Internacional de Dislexia (AID), descreveu o distúrbio como uma dificuldade específica de aprendizagem de origem neurobiológica, caracterizada pela dificuldade do reconhecimento preciso e/ou fluente das palavras e por uma precária habilidade de ortografia e decodificação. Essas dificuldades, em geral, se sucedem em um déficit no componente fonológico da linguagem que frequentemente é imprevisível em relação a outras habilidades cognitivas (IDA, 2002).

Destaca-se ainda, que os resultados secundários decorrentes da DD podem gerar problemas na compreensão da leitura, e uma experiência reduzida de leitura que podem impossibilitar o desenvolvimento do vocabulário e do conhecimento prévio (IDA, 2002). Nesse cenário, cabe citar que os estudantes com dislexia possuem dificuldades ao ler, escrever, embora nem sempre esteja relacionada ao falar (ROMERO; ALONSO; ROMERO, 2020).

Deste modo, indivíduos com DD apresentam afetadas as regiões que se conectam as áreas cerebrais da percepção e da produção da fala, isto é, o progresso motor dos disléxicos acontece de modo diferente dos indivíduos sem o transtorno (SOUZA; SILVA; COUTINHO, 2019). Em síntese, isso ocorre, pois o cérebro de uma pessoa com DD tem dificuldade em identificar ou diferenciar certos tipos de informação, como outros tipos de problemas de aprendizado e atenção. Sendo uma condição que não é superada, apesar de que pode ser melhorada (ROMERO; ALONSO; ROMERO, 2020). 
http://dx.doi.org/10.5902/1984686X54521

Para o delinear dos apontamentos até então apresentados é essencial também uma compreensão de baixo desempenho escolar. Desta maneira, o termo é definido como desempenho, em notas ou tarefas, abaixo de um nível esperado para a idade, habilidade e potencial de um indivíduo (D'ABREU; MARTURANO, 2010). O baixo desempenho escolar resultante da DD produz sérias consequências psicossociais aos alunos com dislexia, por encontrar-se diretamente correlacionada a ansiedade, autoestima, comportamento agressivo, busca por atenção e a outros problemas sociais (ZUPPARDO et al., 2020).

Nesse sentido, altos níveis de ansiedade possui um efeito considerável no baixo desempenho escolar, devido à intermitência do sistema de processamento de informações, por exemplo, a ansiedade elevada no decorrer de uma leitura interfere na memória de curto prazo fonológica (phonological loop), ou seja, na manipulação do material verbal, que exerce um papel fundamental no processamento da linguagem causando a necessidade de ensaios articulatórios, que tributam a capacidade de memória de trabalho, possuindo um papel decisivo em uma série de operações linguísticas, como, a aprendizagem de novas palavras ou a produção e a compreensão da linguagem (NELSON; HARWOOD, 2011).

Nessa perspectiva, uma das variáveis consideradas por Alva (2017), que influenciam nesse cenário, é a autoestima. De acordo com o autor, ela pode ser responsável pelo sucesso ou fracasso escolar. Assim sendo, é primordial entender o nível de autoestima, tal qual, como mencionado acima a ansiedade em estudantes com dislexia, compreendendo suas correlações com o baixo desempenho acadêmico, de modo a executar ações e soluções que melhorem a autoestima dos estudantes.

Para os fins desse trabalho, compreendemos que a DD é uma dificuldade e não uma impossibilidade. Nesse sentido, o presente artigo fundamenta-se no cenário dos estudantes com dislexia, para demonstrar a extrema importância de se discutir sobre o baixo desempenho escolar relacionado aos aspectos emocionais, especificamente, ansiedade e autoestima.

Dessa maneira, este artigo contribui com estudos anteriores para realizá-lo de forma robusta, considerando a literatura científica, explorando os diferentes instrumentos, intervenções e fatores de risco apresentados pelos autores dos artigos analisados que compõe o corpus deste estudo. Sendo assim, o objetivo desse artigo é investigar sobre ansiedade e autoestima associadas ao baixo desempenho escolar em estudantes com dislexia. 
http://dx.doi.org/10.5902/1984686X54521

\section{Método}

Trata-se de uma Revisão Integrativa da Literatura (RIL), que se fundamenta na capacidade de sintetizar o conhecimento científico, proporcionando a análise do conhecimento produzido por outros autores em relação à temática estudada (SANTOS; BRANDÃO; ARAÚJO, 2020).

Para tal, foram utilizadas seis etapas de pesquisa para elaboração desta RIL, como proposto por Mendes, Silveira e Galvão (2008): (1) Seleção da temática abordada, da questão de pesquisa e objetivos; (2) Estabelecimento de critérios de inclusão e exclusão de artigos para busca na literatura; (3) Definição das informações a serem extraídas das publicações selecionadas/categorização desses artigos; (4) Avaliação dos estudos incluídos na revisão integrativa; (5) Interpretação dos resultados; e (6) Apresentação dos resultados obtidos na revisão/síntese do conhecimento.

Na primeira etapa se construiu a questão norteadora utilizando-se a estratégia PICO conforme Santos, Pimenta e Nobre (2007): P - Population/População, I Intervention/Intervenção, C - Comparison/Comparação e O - Outcome/Desfechos esperados. $O$ componente $C$ não se aplica, pois, não se tenciona fazer comparações entre as pesquisas com estudantes com dislexia.

Nesse sentido, para direcionar este estudo, a estratégia PICO delineou-se da seguinte forma: P - estudantes com dislexia, I - instrumentos utilizados para identificar os níveis de ansiedade e autoestima, C - não se aplica e $\mathrm{O}$ - conhecer as implicações do baixo desempenho escolar no desenvolvimento de ansiedade e sua influência na autoestima. Estabeleceu-se, assim, a seguinte questão norteadora: "Quais são as implicações do baixo desempenho escolar no desenvolvimento de ansiedade e sua influência na autoestima dos estudantes com dislexia?".

Com a intenção de pesquisar sobre este panorama, foi realizado um estado da arte sobre a caracterização do transtorno de aprendizagem com ênfase na DD, histórico de ansiedade e autoestima em estudante com DD, fatores relacionados ao baixo desempenho escolar, além de observar publicações em que os autores mencionem futuras perspectivas de intervenções para que as instituições de ensino possam implementar diante desta problemática.

$\mathrm{Na}$ segunda etapa, foram definidos os seguintes critérios de inclusão: estudos que abordassem sobre estudantes com dislexia no ensino fundamental e médio; trabalhos originais (com abordagem qualitativa ou quantitativa), do tipo (estudo de caso, transversal 
http://dx.doi.org/10.5902/1984686X54521

descritivo ou exploratório, caso-controle, e quase-experimental); disponíveis na íntegra; idioma de publicação português, inglês ou espanhol; publicados em periódicos nacionais e internacionais; e que atendessem a pergunta norteadora.

Como critério de exclusão foram retirados: os estudos de revisão; artigos duplicados; pesquisas que envolvem estudantes com dislexia no ensino superior; publicações em repositórios institucionais (monografias, dissertações e teses); livros nacionais e internacionais; e literatura cinzenta (publicações não catalogadas em formato impresso e eletrônico).

Para a seleção dos artigos, (Quadro 1) foram utilizadas as seguintes bases de dados: US Nacional Library of Medicine/Nacional Institutes of Health (MEDLINE/PubMed), Europe PubMed Central (Europe PMC) e Education Resources Information Center (ERIC). O levantamento bibliográfico foi realizado entre os meses de maio a agosto de 2020, nas bases de dados supracitadas anteriormente, a partir do uso dos Descritores em Ciências da Saúde (DeCS) ou Medical Subject Headings (MeSH): ansiedade, autoestima, dislexia de desenvolvimento, dificuldade de desenvolvimento de leitura, educação, estudantes e transtorno da leitura da dislexia do desenvolvimento.

Dessa forma, foi utilizada a seguinte estratégia de busca: \{[Students], [Developmental Dyslexia], [Reading Development Difficulty], [and]\} \{[Anxiety], [Self-esteem], [School performance] [Developmental Dyslexia Reading Disorder], [Developmental Reading Disorder], [Education]\}. Os descritores utilizados foram colocados entre chaves e colchetes para encaminhar a busca dentro dos parâmetros desejados, sendo escolhidos com base nas palavras principais do título dos artigos científicos. A combinação dos descritores e palavras-chave foi realizada por meio dos operadores booleanos AND (combinação restritiva) e OR (combinação aditiva).

O software Mendeley $\AA$ foi utilizado como meio para gerenciamento das referências e importação das publicações nas bases de dados, organizando os artigos em dois grupos identificados como: selecionados para leitura na íntegra e excluídos após leitura de título e resumo.

$\mathrm{Na}$ terceira etapa, foi realizado o processo de elegibilidade das publicações analisadas, de acordo com a exposição do procedimento de busca até a seleção final dos artigos, respaldando-se no Preferred Reporting Items for Systematic Review and MetaAnalysis (PRISMA), conforme fluxograma PRISMA Flow Diagram apresentado na Figura 1 (MOHER et al., 2009). 
http://dx.doi.org/10.5902/1984686X54521

Figura 1 - Estudos selecionados para a RIL, com base no PRISMA Flow Diagram

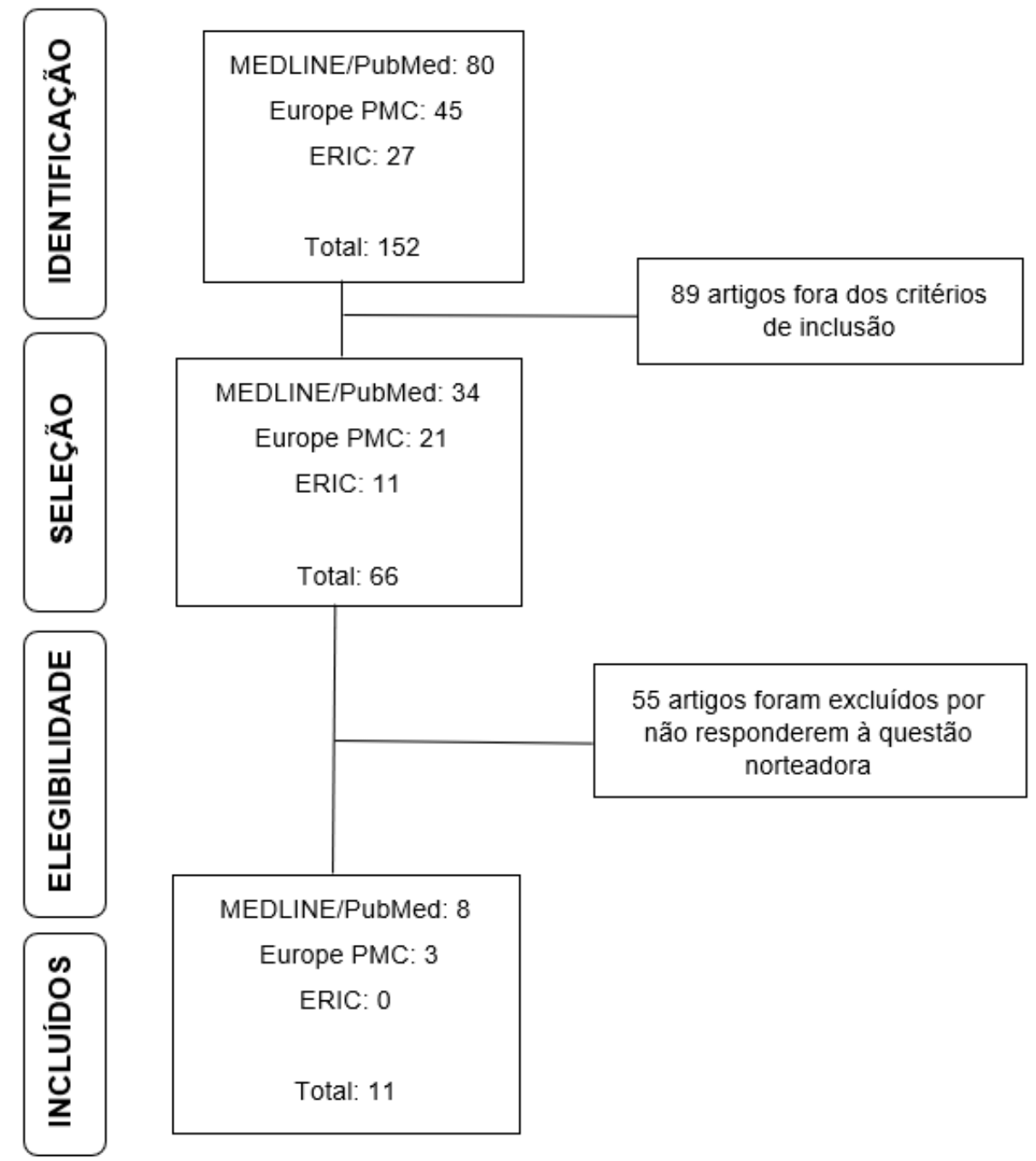

Fonte: Autores (2020) adaptado de Moher et al. (2009).

\section{Resultados}

Os artigos encontrados e utilizados nesta RIL foram publicados no intervalo de 2009 a 2020. A maior concentração de publicações localiza-se nos anos de 2012, 2014, 2016 e 2017, com 2 estudos respectivamente. Sobre o tipo de educação, foi mais preponderante o ensino fundamental com 10 artigos, acompanhado por 1 estudo que abrangeu o ensino médio. Em relação ao país de origem do estudo, 3 são provenientes da Itália e 2 da Espanha. Os demais países (Brasil, Estados Unidos, Alemanha, Inglaterra e Irã) apresentam um estudo incluído, cada um. Não foi encontrado nenhum trabalho que avaliasse ansiedade e autoestima, associadas ao baixo desempenho escolar em 
http://dx.doi.org/10.5902/1984686X54521

estudantes com dislexia, em língua portuguesa, o que nos leva à indagação de que é um problema que não pode ser ignorado em nosso país.

$\mathrm{Na}$ quarta etapa, estes estudos foram lidos na íntegra para a coleta de dados. Tal procedimento buscou identificar: (1) dados gerais dos artigos como título, autor(es), ano, país da publicação e nível de evidência; (2) estrutura das pesquisas como delineamento do estudo, participantes, população estudada, locais em que foi realizada a pesquisa, instrumentos, intervenções para identificação de ansiedade e autoestima; (3) conteúdo dos artigos como objetivo do estudo, caracterização quanto a prevalência de ansiedade e autoestima, relacionada ao baixo desempenho escolar em cada pesquisa, as comorbidades associadas, principais resultados, discussões e considerações.

$\mathrm{Na}$ quinta etapa, após a coleta de dados, foi realizada a interpretação dos resultados. E, por fim, na sexta etapa foi executada a análise e síntese dos resultados encontrados na revisão e dispostos no Quadro 1. Os estudos foram caracterizados através de uma análise bibliométrica, conforme o código de identificação, título dos estudos, autor(es), ano de publicação, país da publicação, objetivos e amostra.

Quadro 1 - Análise bibliométrica dos estudos, segundo o código, título, autor(es), ano, país da publicação, objetivos e amostra

(continua)

\begin{tabular}{|c|c|}
\hline $\begin{array}{c}\text { Código/título/autor(es)/ano/país da } \\
\text { publicação }\end{array}$ & Objetivos/amostra \\
\hline $\begin{array}{l}\text { (E1) • Intervención cognitivo-conductual en un } \\
\text { caso de ansiedad ante la lectura en público: } \\
\text { mejora de la fluidez y comprensión/Escolar- } \\
\text { Llamazares e Serrano-Pintado/2019/Espanha }\end{array}$ & $\begin{array}{c}\text {-Diminuir a ansiedade manifestada quando o } \\
\text { paciente enfrentava a leitura em público e, assim, } \\
\text { melhorar a fluência em leitura e compreensão/1 } \\
\text { estudante com DA }\end{array}$ \\
\hline $\begin{array}{c}\text { (E2) • Anxiety and Reading Difficulties in Early } \\
\text { Elementary School: Evidence for } \\
\text { Unidirectional- or Bi-Directional } \\
\text { Relations?/Grills-Taquechel et } \\
\text { al./2012/Estados Unidos }\end{array}$ & $\begin{array}{c}\text {-Examinar os modelos concorrentes das influências } \\
\text { bidirecionais da ansiedade e realização de } \\
\text { leitura/153 estudantes com DA }\end{array}$ \\
\hline $\begin{array}{c}\text { (E3) • Ansiedad y dificultades escolares/ } \\
\text { Fernandes et al./2014/Brasil }\end{array}$ & $\begin{array}{c}\text {-O principal objetivo é comparar a ansiedade de } \\
\text { crianças que não apresentam problemas escolares } \\
\text { com crianças encaminhadas à clínica psicológica } \\
\text { devido a queixas relacionadas à escola, levando } \\
\text { em consideração o efeito da idade/117 estudantes, } \\
\text { divididos em dois grupos: (1) } \\
37 \text { estudantes com DA; (2) } 80 \text { estudantes sem DA }\end{array}$ \\
\hline $\begin{array}{c}\text { (E4) • Depression, Anxiety at School and Self- } \\
\text { Esteem in Children with Learning } \\
\text { Disabilities/Alesi, Rappo e Pepi/2014/Itália }\end{array}$ & $\begin{array}{l}\text {-Comparar os níveis de depressão, ansiedade na } \\
\text { escola e autoestima em crianças com dificuldades } \\
\text { de aprendizagem, deficiências matemáticas e um } \\
\text { grupo de controle que demonstrou aprendizagem } \\
\text { típica/132 estudantes, divididos em três grupos: (1) } \\
33 \text { estudantes com DA em matemática; (2) } 37 \\
\text { estudantes com dificuldade específica de } \\
\text { aprendizagem; (3) } 62 \text { estudantes sem DA }\end{array}$ \\
\hline
\end{tabular}


http://dx.doi.org/10.5902/1984686X54521

Quadro 1 - Análise bibliométrica dos estudos, segundo o código, título, autor(es), ano, país da publicação, objetivos e amostra

(conclusão)

(E5) • Análisis de la relación entre competencias emocionales, autoestima, clima de aula, rendimiento académico y nivel de bienestar en educación primaria/Morente et al./2017/Espanha

(E6) -Dyslexia and Psycho-social Functioning

An Exploratory Study of the Role of Selfesteem and Understanding/Terras, Thompson e Minnis/2009/Inglaterra

(E7) - Specific Learning Disorders: A Look Inside Children's and Parents' Psychological Well-Being and Relationships/Bonifacci et al./2016/Itália

(E8) - Secondary symptoms of dyslexia: a comparison of self-esteem and anxiety profiles of children with and without dyslexia/Novita/2016/Alemanha

(E9) - Delimitando el perfil emotivo-conductual en niños y Adolescentes con Dislexial Zuppardo, Serrano e Pirrone/2017/Itália

(E10) $\cdot$ The effectiveness of self-instruction technique on improvement of reading performance and reduction of Anxiety in primary school students with Dyslexia/Haddadian et al./2012//rã

(E11) - Las repercusiones de la Dislexia en la Autoestima, en el Comportamiento Socioemocional y en la Ansiedad en Escolares/ Zuppardo et al./2020/Itália
- Examinar a relação entre as variáveis de autoestima, clima de sala de aula, nível de bemestar (avaliado com a escala estado de ansiedade), desempenho acadêmico e habilidades emocionais (consciência emocional, regulação emocional, autonomia emocional, competência e habilidades sociais) vida e bem-estar) em uma amostra de alunos do ensino fundamental/574 estudantes

- Examinar a relação entre dislexia, autoestima e ajuste psicossocial/68 estudantes com dislexia.

-Avaliar os fatores psicológicos, perfis de pais e filhos, além das relações entre suas medidas $/ 68$ tríades compostas por pai, mãe e filho(a), divididas em dois grupos: (1) 34 famílias com crianças com diagnóstico de Distúrbios de Aprendizagem (dislexia, disgrafia, disortografia e discalculia); (2) 34 famílias com crianças sem Distúrbios de Aprendizagem.

- Comparar perfis de ansiedade e autoestima de crianças com e sem dislexia/124 estudantes, divididos em dois grupos: (1) 60 estudantes com dislexia; (2) 60 estudantes sem dislexia - Examinar as consequências emocionais e 0 comportamento relacionado ao fracasso escolar de crianças e adolescentes com dislexia, em comparação com estudantes sem dificuldades de aprendizagem $/ 35$ estudantes, divididos em dois grupos: (1) 25 estudantes com dislexia; (2) 10 estudantes sem dislexia

- Examinar o efeito da técnica de autoinstrução na melhoria do desempenho de leitura e redução dos sintomas de ansiedade em alunos do ensino

fundamental com dislexia da cidade de Arak/32 estudantes, divididos em três grupos: (1) 16 estudantes com dislexia; (2) 16 estudantes com TA; (3) 16 estudantes sem dislexia e TA

-Analisar as características emocionais e comportamentais dos alunos com dislexia em idade escolar em relação aos colegas, sem dificuldades em aprender a ler e escrever. Além disso, pretende-se revelar em quais aspectos específicos (dimensões afetivo-comportamentais) os problemas se manifestam/66 estudantes, divididos em dois grupos: (1) 41 estudantes com dislexia; (2) 25 estudantes sem dislexia

Legenda: Dificuldade de Aprendizagem - DA; Transtorno de Ansiedade - TA.

Fonte: Autores (2020).

De modo geral, os estudos buscaram compreender as relações entre a ansiedade e autoestima em estudantes com dislexia, procurando conhecer sua associação com o baixo 
http://dx.doi.org/10.5902/1984686X54521

desempenho escolar. O Quadro 2 apresenta o código de identificação, instrumentos, delineamento do estudo e os principais resultados encontrados nos estudos que compõem a amostra desta revisão.

Quadro 2 - Quadro-síntese com o código, instrumentos, delineamento do estudo e principais resultados dos artigos desta revisão

(continua)

\begin{tabular}{c}
\hline $\begin{array}{c}\text { Código/instrumentos/delineamento do } \\
\text { estudo }\end{array}$ \\
\hline (E1) •Inventario de Ansiedad ante los \\
Exámene (IAE); El Cuestionario de \\
Interferencia Cognitiva (IC a/b)/Qualitativo, \\
estudo de caso \\
(E2) •Multidimensional Anxiety Scale for \\
Children, (MASC); The Woodcock-Johnson \\
Psycho Educational Test Battery-III, (WJBR); \\
The Continuous Monitoring of Early Reading \\
Skills (CMERS)/Quantitativo, transversal \\
descritivo
\end{tabular}

(E3) •Inventário de ansiedade escolar (IAE)/Quantitativo, caso-controle

(E4) -Raven's Progressive Matrices (RPM);

- The Self Administrated Psychiatric Scales for Children and Adolescents (SAFA); Subescala autoestima escolar/Quantitativo, caso-controle

(E5) • Questionário de desenvolvimento emocional; Evaluación de la Autoestima en

Educación Primaria (AEP); Cuestionario

Clima Social Aula (CES); Stait-Trait Anxiety Inventory for Children (STAIC)/Quantitativo, transversal descritivo

(E6) • Self Profile for Children (SPPC);

Strengths and Difficulties Questionnaire

(SDQ); Understanding and Perceived Impact of Dyslexia Scale (UPIDS)/Quantitativo, transversal exploratório

(E7) •Adult Reading History QuestionnaireRevised (ARHQ-R); Parenting Stress Index (PSI); Symptom Checklist-90-R, SCL-90-R); Parenting Scale (PS); Test of Anxiety and Depression (TAD)/Quantitativo, caso-controle

(E8) -Self-Esteem Test for Children and

Adolescents (ALS); Spence Children's Anxiety Scale (SCAS); Salzburg Reading and Writing Test (SLRT); Culture Fair Intelligence Test-20 Revision, (CFIT)/Quantitativo, casocontrole

(E9) •TMA; MASC/Quantitativo, casocontrole

\section{Principais resultados}

-O tratamento foi eficiente na redução da ansiedade produzido por ler em voz alta na frente dos colegas e professores, além de melhora na habilidade de leitura dos estudantes.

- Os estudantes com baixo desempenho escolar estão em risco de dificuldades subsequentes relacionadas à ansiedade;

-Verificaram-se, através do CMERS, níveis altos de ansiedade de separação.

-As crianças encaminhadas para a clínica de Psicologia apresentaram maior ansiedade do que as demais crianças nos itens relacionados ao medo excessivo e sintomas fisiológicos.

- Os estudantes com DAM e DEA relataram mais sintomas de ansiedade escolar do que seus colegas com níveis típicos de aprendizagem.

-As competências emocionais quanto à autoestima são preditores para os níveis de ansiedade; - O desenvolvimento da inteligência emocional é crucial para o bem-estar psicológico dos estudantes com dislexia.

-Evidenciaram-se dificuldades socioemocionais entre os estudantes com dislexia.

- Os pais de crianças com DA apresentam sofrimento emocional, como também pior histórico de leitura em relação os pais de crianças sem DA;

-As famílias de crianças com DA identificaram seus filhos como mais ansiosos.

-Estudantes com dislexia apresentam maior ansiedade e baixa autoestima no ambiente escolar em comparação com estudantes sem dificuldades semelhantes.

-É fundamental considerar as competências socioemocionais e de aprendizagem na dislexia, uma vez que se busca uma melhoria no desempenho escolar e no bem-estar geral. 
http://dx.doi.org/10.5902/1984686X54521

Quadro 2 - Quadro-síntese com o código, instrumentos, delineamento do estudo e principais resultados dos artigos desta revisão

(conclusão)

\begin{tabular}{|c|c|}
\hline $\begin{array}{c}\text { (E10) } \text {-Wechsler Intelligence Scale for } \\
\begin{array}{c}\text { Children; Reading disorder test; Excitement } \\
\text { disorder test/Quantitativo, quase- } \\
\text { experimental e correlacional }\end{array}\end{array}$ & $\begin{array}{c}\cdot \text { A técnica de autoinstrução tem efeito expressivo na } \\
\text { melhoria do desempenho da leitura, como também na } \\
\text { diminuição de sintomas relacionados à ansiedade em } \\
\text { estudantes com dislexia. }\end{array}$ \\
\hline $\begin{array}{c}\text { (E11) } \cdot \text { TMA; MASC; Child Behavior Check } \\
\text { List (CBCL)/Quantitativo, caso-controle }\end{array}$ & $\begin{array}{c}\text { O perfil emocional-comportamental dos estudantes } \\
\text { com dislexia é concebido pela baixa autoestima e } \\
\text { maior ansiedade em relação aos fatores emocionais, } \\
\text { sendo que facilitam o surgimento de problemas de } \\
\text { ordem comportamental. }\end{array}$ \\
\hline
\end{tabular}

Legenda: Dificuldade de Aprendizagem - DA; Deficiências de Aprendizagem na Matemática - DAM; Dificuldades Específicas de Aprendizagem - DEA.

Fonte: Autores (2020).

Em sequência, foi realizada uma análise qualitativa dos estudos, no qual emergiram duas grandes categorias, compostas por algumas temáticas específicas. Tais temáticas são: intervenções, avaliações, rendimento escolar, saúde mental, preditores psicológicos, comorbidades e qualidade de vida. Sendo identificadas e organizadas a partir do agrupamento das seguintes categorias: 1) Estudos sobre a relação entre ansiedade e o baixo desempenho escolar em estudantes com dislexia (E1, E2, E3, E4, E7, E10); e 2) Estudos sobre a relação entre autoestima e o baixo desempenho escolar em estudantes com dislexia (E5, E6, E8, E9, E11).

\section{Categoria 1 - Estudos sobre a relação entre ansiedade e o baixo desempenho escolar em estudantes com dislexia}

$\mathrm{Na}$ categoria 1, a intervenção encontrada na pesquisa de Escolar-Llamazares e Serrano-Pintado (2019) foi realizada por meio de 24 encontros, com base nas técnicas da Terapia Cognitivo-Comportamental (TCC), com frequência de uma a duas vezes por semana e com média de 60 minutos de duração. Os autores avaliaram a ansiedade ao ler em público, evidenciando-se que se torna um problema gerador de baixo desempenho escolar para 0 estudante que sofre com DD. Além disso, o estudo evidenciou que cerca de $20 \%$ dos estudantes com dificuldades de aprendizagem desenvolvem problemas de ansiedade ao ler.

Tal fato justifica a importância da análise dos resultados obtidos na avaliação prétratamento, que apontaram a presença de traços psicológicos preditores de ansiedade no estudante, relacionado aos seguintes eventos: ler em sala de aula; falar na frente dos pais; desejo de fugir das situações; avaliar negativamente as situações; preocupação com reações 
http://dx.doi.org/10.5902/1984686X54521

somáticas; e o pensamento que nunca será nada na vida se não aprender a ler. Este indivíduo considera que suas fraquezas, quando expostas para estranhos, podem levá-lo à possível ridicularização pelos seus colegas por conta dos erros cometidos (ESCOLARLLAMAZARES; SERRANO-PINTADO, 2019).

Nessa direção, salienta-se que a ansiedade pode se manifestar na forma de um comportamento social inapropriado, como agressão, apatia, entre outros. Em tal cenário, foi demonstrada a efetividade das técnicas da TCC, no qual se observou uma redução da ansiedade produzida por evento aversivo, como, ler em voz alta na frente do professor e dos colegas, como também foi perceptível uma melhoria na sua habilidade de leitura e escrita (ESCOLAR-LLAMAZARES; SERRANO-PINTADO, 2019).

A pesquisa de Grills-Taquechel et al. (2012) foi conduzida como um projeto-piloto avaliando os níveis de ansiedade e monitorando as competências de leitura dos estudantes. Os resultados obtidos, através do instrumento aplicado para mensurar a ansiedade de separação, demostrou a elevação de sintomas ansiogênicos em estudantes que apresentam baixo desempenho acadêmico. Os estudantes apresentaram níveis altos de estresse durante situações, como, dificuldade ao realizar atividades em sala de aula, pressão dos exercícios semelhantes à leitura e pensar que os pais podem se separar.

Grills-Taquechel et al. (2012) verificaram, em seu estudo, que os sintomas de ansiedade de separação comumente aumentam ou são provocados em alunos que sofrem transições, já que enfrentam eventos estressores. Este é o caso dos estudantes com dislexia que participaram da pesquisa, que há pouco tempo passaram para a primeira série do ensino fundamental e estavam tendo como preditor de estresse às situações de baixo desempenho escolar.

O estudo de Fernandes et al. (2014) apontou que a falta de estratégias de aprendizagem está associada ao baixo desempenho escolar e o aumento da ansiedade. Assim, observa-se que os estudantes com dificuldades de aprendizagem, que foram encaminhados a um serviço de clínica psicológica, apresentaram maior predisposição ao desenvolvimento de traços de ansiedade do que os demais estudantes nos itens referentes ao medo excessivo e os sintomas fisiológicos. Uma vez que se considera o surgimento de medo durante uma avaliação de desempenho escolar.

Fernandes et al. (2014) utilizaram em sua pesquisa um instrumento que avaliou traços de ansiedade em estudantes com dislexia, percebendo-se que as situações sociais são os maiores preditores para o surgimento do sentimento de medo e insegurança durante uma 
http://dx.doi.org/10.5902/1984686X54521

avaliação escolar. Destaca-se, ainda, que estes estudantes enfrentam dificuldades de interação com o professor e os colegas de classe, interferindo consideravelmente na qualidade de vida desses alunos.

O trabalho de Alesi, Rappo e Pepi (2014) selecionou estudantes por meio das notas obtidas através da avaliação das dificuldades de aprendizagem. No estudo, foi notável a diferença entre os perfis de aprendizagem de cada estudante em relação aos níveis de ansiedade apresentados. Os alunos pertencentes aos grupos DAM e DEA apresentaram maiores sintomas de ansiedade em relação aos seus colegas com níveis típicos de aprendizagem. Este quadro se deve aos seguintes fatores: dificuldade ao ler na sala de aula; pensar que cometerá diversas falhas ao ler; dificuldade na disciplina matemática; comportamento de esquiva; desejo de fugir das situações aversivas; e presença constante da sensação de fracasso.

Outro ponto relevante é o fato que o perfil emocional desadaptativo e a presença de dificuldade de aprendizagem durante a infância tornam-se um significativo preditor psicológico para o surgimento de ansiedade. As dificuldades de aprendizagem elevam a percepção negativa dos estudantes com dislexia, sobre suas aptidões e intensificam os efeitos negativos, tais como, o humor deprimido, a redução no prazer e interesse, irritabilidade, fadiga e dificuldade de concentração (ALESI; RAPPO; PEPI, 2014).

Bonifacci et al. (2016) evidenciaram, em sua pesquisa, que tanto os estudantes com dislexia quanto seus pais apresentam escores semelhantes para a ansiedade. Nessa mesma direção, os autores observaram que as manifestações de ansiedade nos pais e filhos eram associadas às relações parentais socioafetivas. Ainda, destaca-se o domínio da autoestima como principal preditor para as manifestações de sintomas ansiogênicos nestes indivíduos.

Ainda, segundo os autores, é preciso destacar que as mães frequentemente participam da dinâmica escolar de seus filhos, interagindo com os professores e os demais funcionários da escola. Tornando-se importante levar em consideração a ligação psicoemocional das mães em todo o processo comunicativo destinado à avaliação e intervenções educacionais de seus filhos (BONIFACCI et al., 2016).

No estudo de Haddadian et al. (2012), os participantes foram divididos em dois grupos, um experimental e outro controle. Sendo que para os estudantes do primeiro grupo foram utilizadas técnica de autoinstrução baseadas na TCC, no decorrer de 12 sessões realizadas. Deste modo, a avaliação dos estudantes teve como objetivo obter os diagnósticos de DD e transtorno de ansiedade. 
http://dx.doi.org/10.5902/1984686X54521

Na produção analisada, verificou-se que os estudantes com dislexia apresentavam fatores preditores de ansiedade, como, baixo desempenho escolar, pouco domínio de leitura, medo constante de errar ao tentar ler e a presença de estresse. Neste aspecto, outro fator que merece atenção é o fato que a partir das técnicas da TCC foi possível melhorar significativamente o desempenho de leitura dos estudantes, como também diminuir os níveis de ansiedade (HADDADIAN et al., 2012).

\section{Categoria 2 - Estudos sobre a relação entre autoestima e o baixo desempenho escolar em estudantes com dislexia}

Nessa categoria, o estudo de Morente et al. (2017) destacou que quando os estudantes com dislexia vivenciam situações geradoras de baixo desempenho escolar, tendem a apresentar frustrações decorrentes das atividades difíceis de serem realizadas. Com isso, surge o sentimento de raiva para àqueles que parecem adquirir facilmente aptidões que são consideradas para estes indivíduos como complexas, resultando em insegurança e baixa autoestima.

Além disto, pôde-se observar que tanto as emoções quanto a autoestima e o clima social da sala de aula são importantes preditores psicológicos para o surgimento da ansiedade em estudantes com dislexia. Nessa direção, é possível afirmar que o desenvolvimento das competências emocionais na Educação é uma das recomendações mais nítidas em termos de melhoria do bem-estar, proporcionando uma melhoria na qualidade de vida destes indivíduos (MORENTE et al., 2017).

No estudo realizado por Terras, Thompson e Minnis (2009), foi apontado que os níveis de autoestima estão correlacionados ao baixo desempenho escolar; estando diretamente associados aos altos níveis de sintomas ansiogênicos e problemas nas relações interpessoais no espaço da sala de aula. Diante destes fatores, percebem-se, também, possíveis sinais da presença de comorbidade relacionada ao transtorno de personalidade antissocial nos estudantes com dislexia.

Na pesquisa de Novita (2016), foi possível evidenciar que os estudantes com e sem dislexia apresentam perfis equivalentes de ansiedade e autoestima em geral. Contudo, os estudantes com dislexia apresentaram eventos preditores de ansiedade, como, a presença de estresse, o sentimento de medo ao ler, o receio constante de não conseguir realizar a leitura e a dificuldade de estabelecimentos de relações interpessoais. 
http://dx.doi.org/10.5902/1984686X54521

Zuppardo, Serrano e Pirrone (2017) demonstram, em seu estudo, que os estudantes com dislexia apresentam dificuldade no relacionamento interpessoal/familiar/social, apresentando sentimento constante de desamparo pelo baixo desempenho escolar, provocando ainda, o surgimento de comportamentos agressivos em sala de aula. Todos estes fatores são preditores para a presença de uma menor autoestima em relação aos seus colegas sem dislexia. Cabe ainda ressaltar que diversos aspectos compõem a dimensão que envolve a autoestima, como, por exemplo, a concorrência em sala de aula e o sucesso escolar.

Outro tópico levantado no estudo de Zuppardo, Serrano e Pirrone (2017), foram os problemas de ordem psicopatológica, ao compreender que os estudantes com dislexia possuem maior propensão de apresentar comportamentos desta conjectura, ao se comparar com os estudantes sem dislexia. Desta forma, os problemas seriam de natureza interna, especificamente, manifestando-se a partir de traços psicológicos preditores de ansiedade.

É oportuno enfatizar que os estudantes com dislexia apresentam dificuldade nas relações interpessoais com a presença dos sentimentos de rejeição, humilhação e medo ao realizar atividades em público, além do receio em demostrar suas dificuldades na frente do professor e colegas de sala. Os autores observam que este conjunto de fatores se tornam significativos preditores para o surgimento de problemas de comportamentos externalizantes associados ao baixo desempenho escolar nesses indivíduos (ZUPPARDO et al., 2020).

Retornando aos dados da pesquisa, percebeu-se que o perfil emocionalcomportamental dos estudantes com dislexia demostra que eles possuem uma imagem negativa de si mesmos, resultado da combinação de sua baixa autoestima em várias dimensões, tanto acadêmica quanto pessoal e familiar. Estes estudantes tendem a apresentar comportamentos agressivos e comorbidades relacionadas ao transtorno de ansiedade social ou fobia social, quando comparados aos alunos sem dislexia na execução de atividades em público (ZUPPARDO et al., 2020).

\section{Discussão}

Em linhas gerais, a partir desta revisão, pode-se verificar que a ansiedade e autoestima em estudantes com dislexia tem sido um fenômeno pouco estudado. Isto porque, os resultados deste estudo revelam que poucos estudos a definem e exibem como compreender suas manifestações relacionadas ao baixo desempenho escolar e quais são os preditores psicológicos que podem favorecê-la. Devido à imaturidade 
conceitual do fenômeno na área de conhecimento da Psicologia, grande parte dos estudos incluídos na amostra discute a ansiedade como uma possível consequência do baixo desempenho escolar. Já a autoestima é retratada como uma dimensão interpessoal e emocional vulnerável em indivíduos com dislexia.

Como pôde ser anteriormente observado, os resultados evidenciados por esta pesquisa reforçam a necessidade da implementação de programas de prevenção/intervenção para auxiliar os estudantes com dislexia, presente em 3 artigos (ESCOLAR-LLAMAZARES; SERRANO-PINTADO, 2019; GRILLS-TAQUECHEL et al., 2012; HADDADIAN et al., 2012). Os autores defendem o tratamento através da TCC na melhoria do desempenho de leitura e na redução dos sintomas ansiogênicos. Também, ressaltam a eficácia da técnica de autoinstrução, que consiste em "falar consigo mesmo", com o intuito de colaborar no controle do próprio comportamento. Por sua vez, permite que o estudante tenha uma atitude reflexiva e seja autônomo frente à resolução de atividades e propicie, assim, a interiorização dos processos mentais.

Corroborando com os autores acima mencionados, Assunção et al. (2020) destacam que a TCC detém de um aparato metodológico direto e objetivo, que trabalha demandas atuais, que encontrem-se provocando prejuízo na vida do indivíduo. Tornando-se a abordagem mais indicada no tratamento de sintomas de ansiedade ou de transtornos.

Outro aspecto evidenciado é o baixo desempenho escolar associado à ansiedade. Com a análise dos estudos, percebe-se que 3 artigos contribuíram sobre esta questão, (FERNANDES et al., 2014; ALESI; RAPPO; PEPI, 2014; BONIFACCl et al.,2016). Entende-se que a gestão dos estudantes com dislexia pela escola é lenta, bem como, normalmente é realizada quando o aluno já apresenta diversos sinais relacionados tanto ao processo de alfabetização quanto aos conteúdos escolares. De modo que, com o passar do tempo, os resultados negativos ocasionam o baixo desempenho escolar e sintomas de ansiedade correlacionado ao fracasso em executar as atividades em sala de aula.

Nessas situações, os autores (FERNANDES et al., 2014; ALESI; RAPPO; PEPI, 2014; BONIFACCl et al.,2016) propõem a implementação de programas baseados em estratégias de enfrentamento e solução de problemas, ressaltando-se a necessidade de estabelecer um treinamento dos professores pelos psicólogos. Já é reconhecido que as intervenções educacionais e psicológicas realizadas em conjunto são eficientes para 
http://dx.doi.org/10.5902/1984686X54521

redução das estratégias de desadaptação cognitiva em alunos com dislexia. Assim, há o aprimoramento e a melhoria das relações interpessoais na escola.

Ainda nesse sentido, os autores Carroll e lles (2006) destacaram que é extremamente importante identificar os possíveis sinais de DD por meio do acompanhamento pedagógico e terapêutico. Cabe, ainda, acrescentar que é preciso realizar também uma avaliação psicológica, com a intenção de avaliar através de instrumentos previamente validados no Brasil pelo Sistema de Avaliação de Testes Psicológicos (SATEPSI), para que seja possível determinar a função e os diversos processos psicológicos que compõe o indivíduo.

Não se deve esquecer que o aspecto mais significativo da DD é o indivíduo disléxico. Às vezes, há o perigo de somente levar em consideração o diagnóstico ou programa de apoio que busca realizar, esquecendo-se de sua individualidade e reais necessidades. Em suma, qualquer estratégia de identificação e suporte deve colocar o indivíduo no centro do processo, ou seja, ele tem que ser beneficiado da avaliação e do apoio ofertado (ALVA, 2017).

Colocando-se em foco a autoestima, verificou-se que 5 artigos (MORENTE et al., 2017; TERRAS; THOMPSON; MINNIS, 2009; NOVITA, 2016; ZUPPARDO; SERRANO; PIRRONE, 2017; ZUPPARDO et al., 2020) evidenciaram que os estudantes com dislexia geralmente direcionam suas atenções em coisas que sentem que não executam tão bem quanto os seus colegas. Em função disso, os indivíduos com dislexia consideram difícil a identificação de seus talentos e habilidades, podendo surgir a falta de autoconfiança e baixa autoestima. Assim, é necessário o fortalecimento da autoestima e um bom entendimento da DD, que podem ajudar os estudantes a evitarem essas dificuldades.

Antes de prosseguir, é oportuno compartilhar o conceito de autoestima. Conceitualmente, sua colocação é multidimensional e engloba características acadêmicas, emocionais, físicas, sociais entre outras. Dessa maneira, é preciso ser cauteloso ao afirmar que todos os estudantes com dislexia têm problemas associados à autoestima. Independentemente que esses estudantes tenham a tendência de apresentarem uma autoestima menor que os sem dislexia. Contudo, há uma grande variabilidade entre os indivíduos em relação as suas dimensões e os valores do escore (RAINES, 2016; MAIA FILHO; CHAVES; SEIXAS, 2018).

Tal fato justifica a importância de analisar os dois fatores de risco gerais em indivíduos com DD, relacionados ao domínio específico da autoestima e a um tipo 
http://dx.doi.org/10.5902/1984686X54521

específico de ansiedade. Os problemas secundários da DD são relatados como significativos no ambiente escolar, sendo manifestos durante o seu relacionamento com os professores e colegas. Assim, os professores desempenham um papel importante na gestão das atividades em sala de aula (MAHENDRA; MARIN, 2015; NOVITA, 2016).

Tal narrativa emerge que os professores são indivíduos significativos para o estudante e influenciam o modo como ele se vê ou se percebe, pois eles frequentemente Ihes oferecem informações sobre suas aptidões, habilidades, valores, conhecimentos ou ausência deles. Visto que as avaliações que o próprio aluno faz, a partir de suas vivências escolares, podem interferir, positivamente ou negativamente, em seu desenvolvimento. Nessa direção, entende-se que a qualidade do relacionamento professor-aluno interfere no desenvolvimento do conhecimento de si, elaborado pelo estudante (FERRAZ; RISTUM, 2012).

As análises tecidas nesta revisão reforçam que, para que haja uma redução dos problemas de aprendizagem nos estudantes com dislexia, é preciso realizar intervenções da TCC com foco na Educação socioemocional, buscando a elaboração de estratégias de enfrentamento eficientes, que visem 0 ajustamento social destes indivíduos (OLIVEIRA; BZUNECK; RUFINI, 2017; MOTTA; ROMANI, 2019). Há, então, um reconhecimento da importância da assistência psicológica na escola, para que seja possível a realização de ações que trabalham o autoconhecimento nestes sujeitos, tencionado o desenvolvimento das competências emocionais (ARAUJO; LIMA NETO, 2019; SAMPAIO; OLIVEIRA; ARAÚJO, 2020).

\section{Considerações Finais}

A partir dos resultados apresentados nesta revisão, compreende-se que a DD pode, sem dúvidas, ter uma consequência profunda na vida destes indivíduos. Acredita-se que, por meio da falta de conhecimento e o modo como a sociedade se comporta frente a qualquer sujeito diferente pode ser mais prejudicial do que a DD por si só. Esses indivíduos, muitas vezes, são rotulados como preguiçosos, burros, arrogantes, etc.; ocasionando uma intensificação do baixo desempenho escolar, insegurança e a falta de autoestima nestes sujeitos.

Nesta perspectiva, é de extrema relevância citar que o alto índice de ansiedade, a presença de distúrbios emocionais e comportamentais em estudantes com dislexia, tem como principal consequência o acúmulo de muitas estratégias de enfrentamento 
ineficazes, sendo essencial que haja a identificação precoce para que seja possível responder às necessidades destes indivíduos e de suas famílias, através da assistência psicológica e psicopedagógica nas instituições educacionais.

Assim, pode-se entender que é necessário um maior fortalecimento do diálogo entre a Psicologia e a Educação, no sentido de ampliar ações de intervenções, tais como, as técnicas psicológicas e as práticas pedagógicas inclusivas, tendo como foco orientar os estudantes com dislexia no percurso de superação de suas dificuldades de leitura e ajustamento psicossocial. Evidenciou-se que é preciso um melhor gerenciamento das emoções e um fortalecimento da autoestima, para que haja uma melhoria na qualidade de vida desses estudantes. Diante do exposto, ressalta-se que um bom relacionamento interpessoal no espaço da sala de aula reduz, consideravelmente, os conflitos existentes, através da resolução de problemas e ações preventivas.

Ademais, por se tratar de um contexto pouco explorado, a principal limitação deste estudo foi a escassez de pesquisas na literatura nacional que abordassem ansiedade e autoestima em estudantes com dislexia. Apenas sendo encontrado um estudo que abordava a realidade brasileira em seu escopo, contudo, este não foi publicado no país e sim em um periódico estrangeiro. Nesse sentido, foi utilizado estudos no cenário mundial para que fosse possível discorrer sobre a temática desta revisão. Cabe ainda citar o fato de que apenas foi possível encontrar dados desatualizados no banco de Ministério da Educação do Brasil sobre informações estatísticas de indivíduos identificados com dislexia, que frequentam as escolas públicas e particulares, não contemplando o momento presente do país.

Finalmente, este estudo contribui para o corpo de conhecimento dos psicólogos e profissionais da Educação no tocante aos referenciais teóricos que podem colaborar para novas pesquisas e que possam envolver a realidade do Brasil, cuja intenção seja investigar e desenvolver estratégias com a utilização dos recursos psicológicos e ajustamento social frente à DD. Em suma, esta revisão concretiza a necessidade da elaboração de novas pesquisas que possam aludir à necessidade da ampliação de ferramentas pedagógicas que auxiliem o estudante com dislexia no enfrentamento de suas dificuldades em sala de aula. 
http://dx.doi.org/10.5902/1984686X54521

\section{Referências}

ALESI, Marianna; RAPPO, Gaetano; PEPI, Annamaria. Depression, Anxiety at School and Self-Esteem in Children with Learning Disabilities. Journal of Psychological

Abnormalities in Children, v. 3, n. 3, p. 1-8, 2014. Disponível em:

https://www.longdom.org/abstract/depression-anxiety-at-school-and-selfesteem-inchildren-with-learning-disabilities-21235.html. Acesso em: 20 maio 2020.

ALVA, Manuel L. Chilca. Autoestima, hábitos de estudio y rendimiento académico en estudiantes universitarios. Propósitos y Representacionzes, v. 1, n. 5, p. 71-127, 2017. Disponível em: http://dx.doi.org/10.20511/pyr2017.v5n1.145. Acesso em: 22 maio 2020.

ARAUJO, Karlane; LIMA NETO, Jose Melinho de. Em razão de que se educam as crianças: da aprendizagem ou do desempenho escolar? Revista Educação, Psicologia e Interfaces, v. 3, n. 4, p. 83-97, 2019. Disponível em: https://doi.org/10.37444/issn-25945343.v3i4.202. Acesso em: 25 maio 2020.

ASSUNÇÃO, Wildson Cardoso et al. O tratamento de sintomas de ansiedade baseado na música e Terapia Cognitivo-Comportamental. Revista Educação, Psicologia e Interfaces, v. 4, n. 1, p. 127-143, 2020. Disponível em: https://doi.org/10.37444/issn2594-5343.v4i1.195. Acesso em: 28 maio 2020.

BONIFACCI, Paola et al. Specific Learning Disorders: A Look Inside Children's and Parents' Psychological Well-Being and Relationships. Journal of Learning Disabilities, v. 5, n. 49, p. 532-545, 2016. Disponível em: https://doi.org/10.1177/0022219414566681. Acesso em: 1 jun. 2020.

BRASIL. Ministério da Educação. Dislexia - Comissão assegura direito de aprender, 2008. Disponível em: http://portal.mec.gov.br/component/tags/tag/32256. Acesso em: 12 jun. 2020.

CARROLL, Julia; ILES, Jane. An assessment of anxiety levels in dyslexic students in higher education. British Journal of Educational Psychology, v. 3, n. 76, p. 651-662, 2006. Disponível em: https://doi.org/10.1348/000709905x66233. Acesso em: 14 jun. 2020.

ESCOLAR-LLAMAZARES, María Camino; SERRANO-PINTADO, Isabel. Intervención cognitivo-conductual en un caso de ansiedad ante la lectura en público: mejora de la fluidez y comprensión. Revista de Psicología Clínica Con Niños y Adolescentes, v. 2, n. 6, p. 2019-2054, 2019. Disponível em: http://dx.doi.org/10.21134/rpcna.2019.06.2.8. Acesso em: 20 jun. 2020.

D'ABREU, Lylla Cysne Frota; MARTURANO, Edna Maria. Associação entre comportamentos externalizantes e baixo desempenho escolar: Uma revisão de estudos prospectivos e longitudinais. Estudos de Psicologia (Natal), v. 15, n. 1, p. 43-51, 2010. Disponível em: http://dx.doi.org/10.1590/S1413-294X2010000100006. Acesso em: 25 jun. 2020.

FERNANDES, Débora Cecílio et al. Ansiedad y dificultades escolares. International Journal of Developmental and Educational Psychology, v. 5, n. 1, p. 442, 2016. Disponível em: https://doi.org/10.17060/ijodaep.2014.n1.v5.703. Acesso em: 28 jun. 2020. 
http://dx.doi.org/10.5902/1984686X54521

FERRAZ, Rita de Cássia Souza Nascimento; RISTUM, Marilena. A violência psicológica na relação entre professor e aluno com dificuldades de aprendizagem. Psicologia da Educação, v. 34, n. 1, p. 104-126, 2012. Disponível em:

https://revistas.pucsp.br/index.php/psicoeduca/article/view/28045. Acesso em: 30 jun. 2020.

GRILLS-TAQUECHEL, Amie et al. Anxiety and reading difficulties in early elementary school: evidence for unidirectional- or bi-directional relations? Child Psychiatry and Human Development, v. 1, n. 43, p. 35-47, 2012. Disponível em:

https://dx.doi.org/10.1007\%2Fs10578-011-0246-1. Acesso em: 1 jul. 2020.

HADDADIAN, Fatemeh et al. The Effectiveness of Self-instruction Technique on Improvement of Reading Performance and Reduction of Anxiety in Primary School Students with Dyslexia. Procedia - Social and Behavioral Sciences, v. 1, n. 46, p. 5366-5370, 2012. Disponível em: https://doi.org/10.1016/j.sbspro.2012.06.440. Acesso em: 2 jul. 2020.

IDA. International Dyslexia Association. Definition of Dyslexia (2002). Disponível em: https://dyslexiaida.org/definition-of-dyslexia/. Acesso em: 20 jul. 2020.

MAHENDRA, Fénita; MARIN, Angela Helena. Ambiente familiar e desempenho escolar: uma revisão sistemática. Psicologia da Educação, v. 1, n. 40, p. 41-57, 2015Disponível em: http://pepsic.bvsalud.org/scielo.php?script=sci_abstract\&pid=S141469752015000100004\&lng=pt\&nrm=iso. Acesso em: 25 jul. 2020.

MAIA FILHO, Osterne Nonato; CHAVES, Hamilton Viana; SEIXAS, Pablo de Sousa. Por uma educação para a autonomia de sujeitos situados no mundo. Psicologia da Educação, v. 1, n. 46, p. 81-91, 2018. Disponível em:

https://revistas.pucsp.br/index.php/psicoeduca/article/view/39148. Acesso em: 29 jul. 2020.

MENDES, Karina Dal Sasso; SILVEIRA, Renata Cristina de Campos Pereira; GALVÃO, Cristina Maria. Revisão integrativa: método de pesquisa para a incorporação de evidências na saúde e na enfermagem. Texto \& Contexto - Enfermagem, v, 4, n. 17, p. 758-764, 2008. Disponível em: https://doi.org/10.1590/S0104-07072008000400018. Acesso em: 1 maio 2020.

MOHER, David et al. Preferred reporting items for systematic reviews and meta-analyses: The PRISMA statement. PLoS Medicine, v. 7, n. 6, p. 1-6, 2009. Disponível em: https://doi.org/10.1371/journal.pmed.1000097. Acesso em: 1 maio 2020.

MORENTE, Agnes Ros et al. Análisis de la relación entre competencias emocionales, autoestima, clima de aula, rendimiento académico y nivel de bienestar en educación primarias, autoestima, clima de aula, rendimiento académico y nivel de bienestar en educación primaria. Revista Española de Orientación y Psicopedagogía, v. 1, n. 28, p. 8-18, 2017. Disponível em: http://revistas.uned.es/index.php/reop/article/view/19355. Acesso em: 2 ago. 2020.

MOTTA, Pierre Cerveira; ROMANI, Patrícia Fasolo. A Educação Socioemocional e suas implicações no contexto escolar: uma revisão de literatura. Revista Psicologia da Educação, v. 1, n. 49, p. 49-56, 2019. Disponível em: http://dx.doi.org/10.5935/21753520.20190018. Acesso em: 4 ago. 2020. 
NELSON, Jason M.; HARWOOD, Hannah. Learning disabilities and anxiety: a metaanalysis. Journal of Learning Disabilities, v. 1, n. 44, p. 3-17, 2011. Disponível em: https://doi.org/10.1177\%2F0022219409359939. Acesso em: 8 ago. 2020.

NOVITA, Shally. Secondary symptoms of dyslexia: a comparison of self-esteem and anxiety profiles of children with and without dyslexia. European Journal of Special Needs Education, v.2, n. 31, p. 279-288. 2016. Disponível em: https://doi.org/10.1080/08856257.2015.1125694. Acesso em: 12 ago. 2020.

OLIVEIRA, Maria Fernanda Cunha; BZUNECK, José Aloyseo; RUFINI, Sueli Édi. Motivação de adolescentes para leitura: Estudo com a abordagem centrada na pessoa. Revista Psicologia da Educação, v. 2, n. 45, p. 67-76, 2017. Disponível em: http://dx.doi.org/10.5935/2175-3520.20170018. Acesso em: 14 ago. 2020.

RAINES, James. Improving the Self-Esteem and Social Skills of Students with Learning Disabilities. Encyclopedia of Social Work: National Association of Social Workers Press and Orxford University Press, 2016. Disponível em:

https://doi.org/10.1093/acrefore/9780199975839.013.1218. Acesso em: 22 jun. 2020.

ROMERO, María González; ALONSO, Jose María Rabal; ROMERO, José González. Aproximación conceptual a la dislexia en las aulas de Educación primaria. Brazilian Journal of Development, v. 4, n. 6, p. 20157-20165, 2020. Disponível em: https://doi.org/10.34117/bjdv6n4-260. Acesso em: 15 ago. 2020.

SAMPAIO, Joana; OLIVEIRA, Íris M.; ARAÚJO, Alexandra M. Competência emocional como recurso para o envolvimento de adolescentes na escola. Revista Psicologia em Pesquisa, v. 14, n. 1, p. 57-75, 2020. Disponível em: http://dx.doi.org/10.34019/19821247.2020.v14.27646. Acesso em: 16 ago. 2020.

SANTOS, Cristina Mamédio da Costa; PIMENTA, Cibele Andrucioli de Mattos; NOBRE, Moacyr Roberto Cuce. A estratégia PICO para a construção da pergunta de pesquisa e busca de evidências. Revista Latino-Americana de Enfermagem, v. 3, n. 15, p. 508511, 2007. Disponível em: https://doi.org/10.1590/S0104-11692007000300023. Acesso em: 17 ago. 2020.

SANTOS, Stephany da Silva; BRANDÃO, Gisetti Corina Gomes; ARAÚJO, Kleane Maria da Fonseca Azevedo. Social isolation: a look health elderly mental during the COVID-19 pandemic. Research, Society and Development, v. 7, n. 9, p. 1-15, 2020. Disponível em: https://doi.org/10.33448/rsd-v9i7.4244. Acesso em: 18 ago. 2020.

SOUZA, Gustavo de Lima; SILVA, Roseane Maria da Costa; COUTINHO, Diógenes José Gusmão. Dislexia e dislalia: necessidades e possibilidades na prática inclusiva. Brazilian Journal of Development, v. 12, n. 5, p. 32009-32018, 2019. Disponível em: https://doi.org/10.34117/bjdv5n12-281. Acesso em: 19 ago. 2020.

TERRAS, Melody; THOMPSON, Lucy; MINNIS, Helen. Dyslexia and psycho-social functioning: An exploratory study of the role of self-esteem and understanding. Dyslexia, v. 4, n. 15, p. 304-327, 2009. Disponível em: https://doi.org/10.1002/dys.386. Acesso em: 20 ago. 2020. 
ZUPPARDO, Linda; SERRANO, Francisca; PIRRONE, Concetta. Delimitando el perfil emotivo-conductual en niños y adolescentes con dislexia. Revista RETOS XXI Discapacidad y Educación, v.1, n. 1, p. 88-104, 2017. Disponível em:

https://revistas.utp.ac.pa/index.php/retoxxi/article/view/1521. Acesso em 22 ago. 2020.

ZUPPARDO, Linda et al. Las repercusiones de la Dislexia en la Autoestima, en el Comportamiento Socioemocional y en la Ansiedad en Escolares. Psicología Educativa, v. 2, n. 26, p. 175-183, 2020. Disponível em: https://doi.org/10.5093/psed2020a4. Acesso em 22 ago. 2020.

(c) (i) (9)

This work is licensed under a Creative Commons Attribution-NonCommercial 4.0 International (CC BY-NC 4.0) 